\title{
High-Quality Electron Beams from Beam-Driven Plasma Accelerators by Wakefield-Induced Ionization Injection
}

\author{
A. Martinez de la Ossa, J. Grebenyuk, T. Mehrling, L. Schaper, and J. Osterhoff \\ Deutsches Elektronen-Synchrotron DESY, D-22607 Hamburg, Germany
}

(Received 9 September 2013; published 10 December 2013)

\begin{abstract}
We propose a new and simple strategy for controlled ionization-induced trapping of electrons in a beam-driven plasma accelerator. The presented method directly exploits electric wakefields to ionize electrons from a dopant gas and capture them into a well-defined volume of the accelerating and focusing wake phase, leading to high-quality witness bunches. This injection principle is explained by example of three-dimensional particle-in-cell calculations using the code OSIRIS. In these simulations a high-currentdensity electron-beam driver excites plasma waves in the blowout regime inside a fully ionized hydrogen plasma of density $5 \times 10^{17} \mathrm{~cm}^{-3}$. Within an embedded $100 \mu \mathrm{m}$ long plasma column contaminated with neutral helium gas, the wakefields trigger ionization, trapping of a defined fraction of the released electrons, and subsequent acceleration. The hereby generated electron beam features a $1.5 \mathrm{kA}$ peak current, $1.5 \mu \mathrm{m}$ transverse normalized emittance, an uncorrelated energy spread of $0.3 \%$ on a GeV-energy scale, and few femtosecond bunch length.
\end{abstract}

DOI: 10.1103/PhysRevLett.111.245003

Over the last decade, the field of plasma-wakefield acceleration of electrons with field gradients surpassing $10 \mathrm{GV} / \mathrm{m}$ has progressed rapidly, leading to an improvement in the quality of the accelerated beams. In particular, laser-driven wakefield accelerators [1] were advanced significantly. Milestones, such as the realization of quasimonoenergetic electron spectra [2-4], GeV-class beams [5], enhanced stability [6,7], controlled injection techniques for tunability [8-10], and the application of the generated beams to drive compact extreme ultraviolet [11] and $\mathrm{x}$-ray sources [12] validated plasma-based acceleration as a promising technique for future accelerators. Meanwhile, beam-driven plasma wakefield acceleration (PWFA) $[13,14]$ made great progress, culminating in the demonstration of energy doubling of part of the $42 \mathrm{GeV}$ SLAC electron beam [15]. However, the quality of electron bunches extracted from beam-driven schemes lags behind those obtained from laser-plasma accelerators. This may be attributed to a so-far insufficient control over the electron-injection process in PWFA, which has fundamental impact on the initial beam phase-space population and, thus, on the final beam quality.

Several controlled injection techniques for PWFA, such as the external injection of a witness beam [16], magnetically induced injection [17], and laser-triggered ionization injection $[18,19]$, have been proposed, but have not been experimentally verified yet. All these methods demand several elements in the experiment to act in concert to achieve injection into the appropriate wake region, e.g., fs synchronization and $\mu \mathrm{m}$ alignment of a laser to the particle beam, or the generation of an adequate witness beam and its matching into the plasma wakefield [20]. These measures can be technically challenging to implement and may become a source of instabilities, which
PACS numbers: 52.40.Mj, 41.75.Ht, 52.25.Jm, 52.59.Bi

complicates the generation of high-quality beams. An easier approach constitutes the injection of electrons in plasma by means of field-induced ionization of a dopant gas with appropriate ionization potential, e.g., helium (He). This process can be initiated by the radial electric field of the driving beam [21]. However, the lack of control over the event and its sensitivity to initial conditions of the driver microstructure have not yet resulted in the production of qualitatively interesting beams.

In this Letter, we propose a new and straightforward strategy for controlling ionization injection of electrons into beam-driven plasma wakes, which utilizes the wake electric fields only, and thus, provides improved beam quality. This technique exploits the difference in absolute electric-field strength in the blowout regime [22-24] between the accelerating and decelerating regions within the first wakefield bucket to selectively ionize a small volume of a background dopant gas near the phase of maximum acceleration. In this way the production of high-quality, ultrashort $(\sim \mathrm{fs})$, low-emittance $(\sim \mu \mathrm{m})$, multi-GeV-energy electron beams from a relatively simple experimental setup is made possible.

PWFA in the blowout regime uses a relativistic charged particle beam shorter than the plasma wavelength and of higher density than the plasma background. This driver expels plasma electrons from its high-density core, forming a copropagating ion cavity. The electric fields in this cavity or bubble may exceed the cold nonrelativistic wave-breaking limit $E_{0}=\left(m c^{2} / e\right) k_{p}$, where $k_{p}=\sqrt{n_{0} e^{2} / \epsilon_{0} m c^{2}}$ is the plasma wave number, $n_{0}$ the plasma particle density, $\epsilon_{0}$ the vacuum permittivity, $c$ the speed of light, and $m$ and $e$ are the electron mass and charge, respectively. Current accelerator facilities provide 
short $10-50 \mu \mathrm{m}$ rms electron beams with currents of 1-25 kA suitable to operate wakefields in the blowout regime with fields of $\sim 10-100 \mathrm{GV} / \mathrm{m}$ at plasma densities of $\sim 10^{17} \mathrm{~cm}^{-3}$. The amplitude of these wakefields can be sufficient to ionize electrons from a high-ionizationpotential atomic species such as He, and trigger their trapping into a defined phase near the back of the ion cavity. The ionization process caused by static (or slowly varying) electric fields of a strength sufficient to significantly deform the atomic potential barrier can be described by a tunneling probability [25], and has been determined for a number of atomic species [26]. Writing the tunneling-ionization rate according to the theory by Ammosov, Delone, and Krainov (ADK) in an engineering formula yields [27]

$$
\begin{aligned}
W_{\mathrm{ADK}}\left[\mathrm{fs}^{-1}\right] \approx & 1.52 \frac{4^{n^{*}} \xi_{i}[\mathrm{eV}]}{n^{*} \Gamma\left(2 n^{*}\right)}\left(20.5 \frac{\xi_{i}^{3 / 2}[\mathrm{eV}]}{E[\mathrm{GV} / \mathrm{m}]}\right)^{2 n^{*}-1} \\
& \times \exp \left(-6.83 \frac{\xi_{i}^{3 / 2}[\mathrm{eV}]}{E[\mathrm{GV} / \mathrm{m}]}\right)
\end{aligned}
$$

where $\xi_{i}[\mathrm{eV}]$ is the potential energy of the bound electron, $n^{*} \approx 3.69 Z / \xi_{i}^{1 / 2}[\mathrm{eV}]$ is the effective principal quantum number, which depends on the ionization level $Z$, and $E=\sqrt{E_{x}^{2}+E_{y}^{2}+E_{z}^{2}}$ is the absolute electric field acting on the atom. The field $E=E_{\text {ion }}$ for which the ionization rate becomes $W_{\mathrm{ADK}}=0.1 \mathrm{fs}^{-1}$ is in this Letter considered as the ionization threshold. In case of $\mathrm{He}, E_{\mathrm{ion}}^{\mathrm{He}}=93 \mathrm{GV} / \mathrm{m}$ is predicted for the tunneling of the outer electron $(Z=1$, $\left.\xi_{i}=23.6 \mathrm{eV}\right), \quad E_{\mathrm{ion}}^{\mathrm{He}^{+}}=235 \mathrm{GV} / \mathrm{m}$ for the inner one $\left(Z=2, \quad \xi_{i}=54.4 \mathrm{eV}\right)$. The electron dynamics after release by the above mechanism can be addressed considering the Hamiltonian for a single electron $\mathcal{H}(\vec{x}, \vec{P}, t)=$ $\sqrt{\left(m c^{2}\right)^{2}+c^{2}(\vec{P}+e \vec{A})^{2}}-e \Phi$, characterized by the potentials $\Phi(\vec{x}, t)$ and $\vec{A}(\vec{x}, t)$ and the generalized coordinates $\vec{x}$ and $\vec{P}=\vec{p}-e \vec{A}$, where $\vec{p}$ is the electron momentum. In the comoving system of reference with $v_{\mathrm{ph}}$ the phase velocity of the wake and $\zeta=z-v_{\mathrm{ph}} t$, the electromagnetic potentials barely change over time compared to their variation with $\zeta$. Therefore, the quasistatic approximation [28] holds and $\partial_{t}=-v_{\mathrm{ph}} \partial_{\zeta}=-v_{\mathrm{ph}} \partial_{z}$. In this case, provided that $\dot{\mathcal{H}}=\partial_{t} \mathcal{H}=-v_{\mathrm{ph}} \partial_{z} \mathcal{H}=v_{\mathrm{ph}} \dot{P}_{z}$, the quantity $\mathcal{K} \equiv \mathcal{H}-v_{\mathrm{ph}} P_{z}=m c^{2} \gamma-v_{\mathrm{ph}} p_{z}-e \Psi$ is a constant of motion [29]. Here, we have defined the potential $\Psi \equiv$ $\Phi-v_{\mathrm{ph}} A_{z}$ related to the electric and magnetic fields by $E_{z}=-\partial_{z} \Psi$ and $E_{r}-v_{\mathrm{ph}} B_{\phi}=-\partial_{r} \Psi$, while $\gamma$ is the Lorentz factor of the electron. Immediately after ionization, the electron has negligible energy and can be considered at rest, thus $\mathcal{K}_{i}=m c^{2}-e \Psi_{i}$. This electron is trapped into the wake if it follows a phase-space trajectory such that its velocity $v$ reaches $v_{\mathrm{ph}}$. When this happens $\mathcal{K}_{f}=$ $m c^{2} / \gamma_{\mathrm{ph}}-e \Psi_{f}$, and since $\mathcal{K}_{f}=\mathcal{K}_{i}$, a trapping condition in terms of potential difference between the initial $\Psi_{i}$ and trapped $\Psi_{f}$ state may be expressed as $\Delta \Psi \equiv \Psi_{f}-$ $\Psi_{i}=-m c^{2} / e\left(1-\gamma_{\mathrm{ph}}^{-1}\right)$ [21]. For ultrarelativistic drivers $\left(v_{\mathrm{ph}} \rightarrow c\right.$ and $\left.\gamma_{\mathrm{ph}} \rightarrow \infty\right)$, this condition can be written as $\Delta \Psi=-m c^{2} / e$. The initial position of the ionized electron inside the wake determines $\Psi_{i}$, and, consequently, its final trapping position (if any) along the corresponding $\Psi_{f}$ equipotential contour. The necessary trapping condition for electrons ionized inside the first wake period, ahead of the potential minimum $\Psi_{\min }$ at the rear of the ion cavity, is given by $\Psi_{i}>\Psi_{\min }+m c^{2} / e \equiv \Psi_{t}$. The volume of injection is thus determined by the intersection of the volume of ionization $\left(E_{i}>E_{\text {ion }}\right)$ with the volume satisfying the trapping criterion $\left(\Psi_{i}>\Psi_{t}\right)$. Generally, the field configuration in the blowout regime can enable simultaneous ionization and trapping in two regions within the first wave bucket [30]. One is located at the driver-beam position, where the radial electric field induces ionization. This ionization region is sensitive to the oscillating behavior of the beam in the focusing ion column [31] and to fluctuations in the microstructure of its density profile. The second region is located at the rear of the cavity, where the wakefields trigger ionization. In contrast to the front, the fields at the back are more stable in time and depend to a lesser degree on details of the driver-beam profile, and thus, provide a well-defined and controlled region for injection. The injection technique proposed in this Letter is designed to inject electrons only from a narrow phase interval at the back of the cavity, while preventing any direct contribution from the radial electric field of the driver.

To illustrate this method in the following, we consider electron bunches similar to those provided by the FACET facility at SLAC. These beams are approximated by Gaussian longitudinal $\left(\sigma_{z}=14 \mu \mathrm{m}\right)$ and transverse $\left(\sigma_{x}=\sigma_{y}=10 \mu \mathrm{m}\right)$ profiles with peak currents of $23 \mathrm{kA}$, transverse normalized emittances of $\epsilon_{x}=50 \mu \mathrm{m}$ and $\epsilon_{y}=5 \mu \mathrm{m}$, and an energy of $23 \mathrm{GeV}$ with a relative spread of $1 \%$ [16]. These characteristics make them suitable to operate in the blowout regime in a plasma of density $n_{0}=5 \times 10^{17} \mathrm{~cm}^{-3}$, which can be generated using current gas cell technology [32]. As sketched in Fig. 1, a micronozzle [33] fed by a hydrogen-helium mixture with tunable ratio and pressure, is positioned in the vicinity of the gas cell entrance. The gas jet emerging from the nozzle is spatially confined to a diameter of about $L_{\mathrm{He}}=100 \mu \mathrm{m}$, forming a highly localized region in which helium is

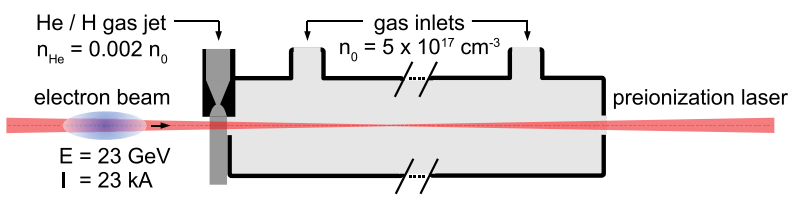

FIG. 1 (color online). Schematic of the plasma-cell setup used in OSIRIS 3D simulations. A thin jet of a neutral $\mathrm{H} / \mathrm{He}$ gas mixture is immersed in a laser preionized hydrogen plasma at $n_{0}$. 
present while maintaining a flat hydrogen density distribution in line of sight of the electron beam. In order to prevent excessive beam loading, we choose a He concentration of $n_{\mathrm{He}}=0.002 n_{0}$. In this setup, the plasma is precreated by a laser pulse coaxial to the electron beam with an intensity of $I_{L} \geq 1.52 \times 10^{14} \mathrm{~W} / \mathrm{cm}^{2}$ capable to fully ionize the hydrogen, but not the helium at $I_{L} \ll 1.14 \times$ $10^{15} \mathrm{~W} / \mathrm{cm}^{2}$. These limits are calculated from $E_{\mathrm{ion}}^{\mathrm{He}}=$ $93 \mathrm{GV} / \mathrm{m}$ and $E_{\mathrm{ion}}^{\mathrm{H}}=34 \mathrm{GV} / \mathrm{m}$, the ionization thresholds for $\mathrm{He}$ and $\mathrm{H}$, respectively. Such intensities are easily realized by 100 -fs scale sub-TW titanium:sapphire lasers which are readily available.

Three-dimensional (3D) simulations of this setup have been performed using the particle-in-cell (PIC) code OSIRIS [34], which is capable of emulating ionization effects using the ADK model [26]. The moving window simulation box dimensions are $18 \times 30 \times 30 k_{p}^{-3}$ with a cell size of $0.036 \times 0.060 \times 0.060 k_{p}^{-3}$. Figure 2 (a) shows the plasma electron density, the driver beam density, and the density of ionized electrons in the central slice ( $y$ vs $\zeta$, at $x=0$ ) of the simulation inside the He region. The magnitude of the electric field [Fig. 2(b)] at the rear of the ion cavity exceeds the threshold for the ionization of helium $E_{\mathrm{ion}}^{\mathrm{He}}$ by far, whereas it is significantly lower in the driver region. To restrict the area of injection to the back of the bubble, the radial space-charge field of the driver $\left|E_{r}\right|$, which is inversely dependent on the transverse beam size, must be less than $E_{\mathrm{ion}}^{\mathrm{He}}$ during passage through the He gas jet. This is ensured by placing the jet at the entrance of the plasma target, well before the beam experiences first compression induced by the focusing ion cavity [31]. The length scale of this transverse focusing is given by the betatron wavelength $\lambda_{\beta}=\sqrt{2 \gamma} \lambda_{p}$ [35], in the considered case $\lambda_{\beta} \approx$ $14 \mathrm{~mm}$. Figure 2(c) shows the probability of ionization $P_{\mathrm{ADK}}(r, \zeta)$ of the He atoms streaming backwards with respect to the wake, obtained by integrating the ionization rate $W_{\mathrm{ADK}}$ in Eq. (1) along $\zeta$. The contours where $P_{\mathrm{ADK}}$ reaches $10 \%$ and $100 \%$ of ionization are included, defining a narrow phase interval $\left\{\zeta_{10}, \zeta_{100}\right\}$ extending to the borders of the bubble, from which $90 \%$ of all potentially trapped He electrons are emerging $\left(\Delta \zeta_{\text {ion }} \equiv \zeta_{10}-\zeta_{100} \approx 3 \mu \mathrm{m}\right)$. Figure 2(d) depicts the trapping potential $\Psi-\Psi_{t}$, where positive values correspond to regions which allow trapping $\left(\Psi>\Psi_{t}\right)$. Equipotential contours are shown in steps of $0.2 \times m c^{2} / e$. The intersection of the volume with high ionization probability and the volume allowing trapping yields the volume from which injected electrons can originate [Fig. 2(a)]. Trapping is also affected by the transverse dynamics of the electron in the plasma wave. Electrons released off axis close to the rear boundary of the cavity may escape before the focusing force pushes them towards a stopping contour near the axis. A sufficient condition for trapping such electrons is fulfilled if they reach a $\Psi_{f}$ contour before entering a defocusing region in straight backwards propagation. Here, the maximum radius

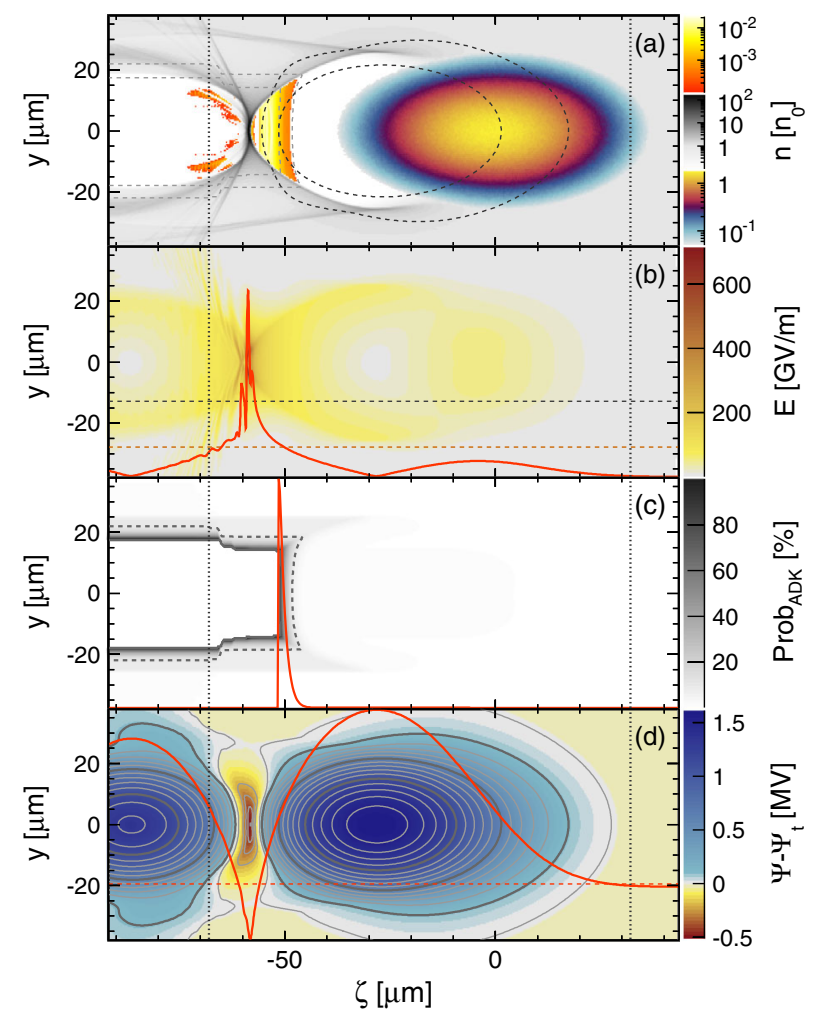

FIG. 2 (color online). Central slices from a 3D OSIRIS simulation depicting the trapping conditions and showing ionization of electrons from a He dopant by wakefields excited by a FACETtype beam in a precreated uniform plasma with a density of $n_{e}=5 \times 10^{17} \mathrm{~cm}^{-3}$. (a) Spatial particle density. Plasma (middle palette), driver beam (bottom palette), and electrons ionized from $\mathrm{He}$ (top palette). (b) Total electric field and on-axis values (solid line). Light (dark) dashed line shows the ionization threshold of the outer (inner) He electron. (c) ADK ionization probability of the first level of $\mathrm{He}$, the contour at $10 \%$ probability (dashed line), and the on-axis values (solid line). (d) The electric potential $\Psi-\Psi_{t}$, its contours in steps of $\Delta \Psi=0.2 \times\left(m c^{2} / e\right)$, and the on-axis values (solid line). Vertical dotted lines show the limits of the He column.

fulfilling this condition is $R_{\max } \approx 12 \mu \mathrm{m}$ [cf. Fig. 2(a)]. This allows for an estimate of the volume of injection $V_{\mathrm{inj}} \simeq \pi R_{\mathrm{max}}^{2} \Delta \zeta_{\mathrm{ion}}$ and hence of the total trapped charge $Q_{\mathrm{He}} \simeq-e n_{\mathrm{He}} \pi R_{\max }^{2} L_{\mathrm{He}}=7.2 \mathrm{pC}$.

Figure 3(a) shows a short $(0.8 \mu \mathrm{mrms})$ bunch of electrons injected from the neutral $\mathrm{He}$ by means of the wakefields in the above discussed simulation. With a total charge of $8.8 \mathrm{pC}$ and a maximum peak current of $1.5 \mathrm{kA}$, the injected beam has been accelerating for $20 \mathrm{~mm}$, positioned at $\left\langle\zeta_{f}\right\rangle=-55.7 \mu \mathrm{m}$, where the longitudinal electric field is $E_{z}\left(\left\langle\zeta_{f}\right\rangle\right) \approx 130 \mathrm{GV} / \mathrm{m}$ [Fig. 3(b)]. Most properties of the trapped bunch can be estimated from the initial phase-space distribution. Trapped electrons with the same initial $\Psi_{i}$ are positioned approximately at the same comoving phase near axis during acceleration, fulfilling $\Psi_{f}\left(\zeta_{f}\right)=\Psi_{i}\left(\zeta_{i}\right)-m c^{2} / e$, and thus will be accelerated by the same field value $E_{z}\left(\zeta_{f}\right)$. However, each 


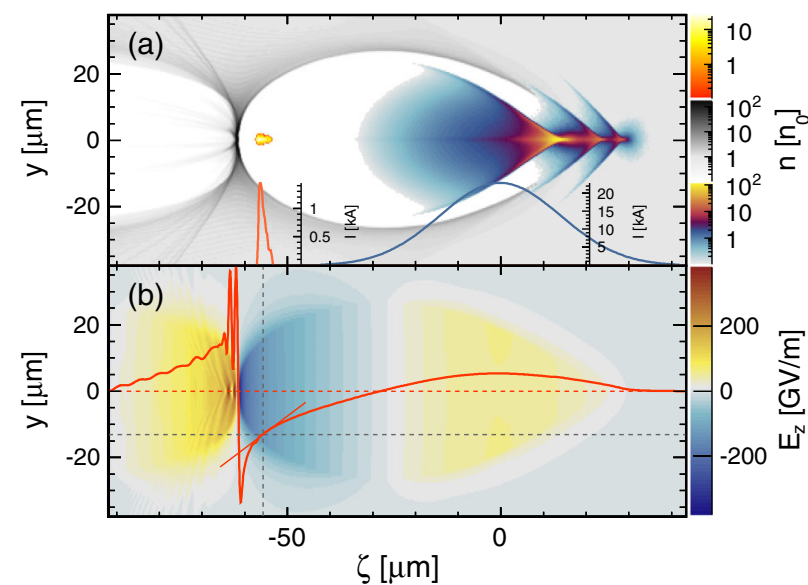

FIG. 3 (color online). PIC simulation after $20 \mathrm{~mm}$ of beam propagation. (a) Spatial particle density as in Fig. 2(a). The curves show currents of the drive beam (right) and injected electrons (left). (b) Longitudinal wakefields.

one of these slices in $\zeta_{f}$ is composed of electrons ionized at different longitudinal positions in the He column, and therefore are accelerated over different times, producing a finite spread in longitudinal momentum in every slice given by $\Delta p_{z}\left(\zeta_{f}\right) \simeq-e E_{z}\left(\zeta_{f}\right) L_{\mathrm{He}}$, which at the average position of the bunch gives $\Delta p_{z}\left(\left\langle\zeta_{f}\right\rangle\right) \approx 13 \mathrm{MeV}$. Moreover, the total relative energy spread is proportional to the variation of $E_{z}$ along the bunch, which in case of negligible beam loading and sufficiently short bunches is approximately given by $\Delta \gamma / \gamma \simeq \partial_{\zeta} E_{z}\left(\left\langle\zeta_{f}\right\rangle\right) / E_{z}\left(\left\langle\zeta_{f}\right\rangle\right) \Delta \zeta_{f}$. From Fig. 3(b), $\partial_{\zeta} E_{z}\left(\left\langle\zeta_{f}\right\rangle\right) \approx 10(\mathrm{GV} / \mathrm{m}) \mu \mathrm{m}^{-1}$, and $\Delta \gamma / \gamma \approx 6 \%$. Electrons belonging to the same $\zeta_{f}$ slice originate from different radial positions along their initial $\Psi_{i}$ contour. Assuming full betatron decoherence for every slice, an upper estimate of the normalized transverse emittance $\epsilon_{y}=\sqrt{\left\langle y^{2}\right\rangle\left\langle p_{y}^{2}\right\rangle-\left\langle y p_{y}\right\rangle^{2}} / m c$ can be given in terms of the initial transverse extent of the slice [30] $\epsilon_{y}=k_{p}\left\langle y_{i}^{2}\right\rangle / 4$. Considering for simplicity, the largest $\Psi_{i}$ contour to be uniformly distributed up to $R_{\max }$, the estimated maximum sliced emittance yields $\epsilon_{y, \max }=k_{p} R_{\max }^{2} / 12 \approx 1.4 \mu \mathrm{m}$.

The properties of the simulated injected bunch after $20 \mathrm{~mm}$ of acceleration are summarized in Fig. 4. The longitudinal phase space [Fig. 4(a)] exhibits linear chirp with an average energy of $\sim 2.6 \mathrm{GeV}$ and a total relative energy spread of $6 \%$ rms. The sliced bunch properties can be seen in detail in Fig. 4(b). The current profile has a maximum at the tail of the bunch of $\sim 1.5 \mathrm{kA}$ and linearly decreases towards its front [Fig. 4(b)]. The relative energy spread $(\sim 0.3 \%)$, and the normalized transverse emittance $(\leq 1.5 \mu \mathrm{m})$ are shown for different slices in $\zeta$, demonstrating an excellent agreement with the analytical calculations given previously. Further acceleration of the captured beam is possible until the driver

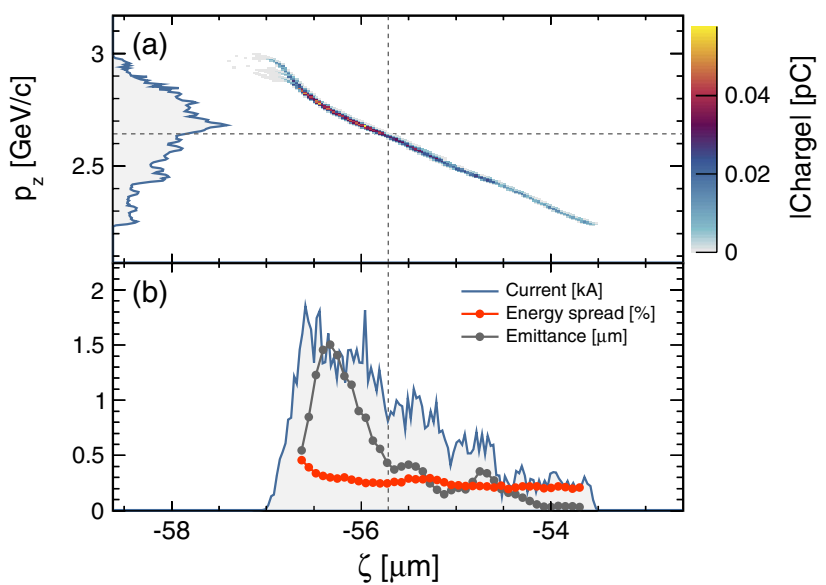

FIG. 4 (color online). Witness bunch properties after $20 \mathrm{~mm}$ of acceleration. (a) shows the absolute charge distribution of the bunch in longitudinal phase space ( $p_{z}$ vs $\zeta$ plane). The projection of this distribution in $p_{z}$ is depicted on the left axis. (b) displays the bunch-current dependence on $\zeta$. The relative energy spread and the transverse emittance are plotted for different longitudinal slices along the bunch.

has exhausted its energy. A simple estimate yields the maximum achievable bunch energy when considering the decelerating gradients experienced by the driver beam of $50 \mathrm{GV} / \mathrm{m}$ [cf. Fig. 3(b)]. This limits the driver propagation distance to $\sim 46 \mathrm{~cm}$, and hence, the maximum energy of the injected beam to $46 \mathrm{GeV}$ assuming an acceleration of the trailing bunch at a continuing rate of $100 \mathrm{GV} / \mathrm{m}$. These values are in agreement with previous experimental observations demonstrating energy doubling [15].

In summary, a new strategy for the injection of electrons in PWFA is proposed and demonstrated using 3D PIC simulations. The described method leads to a controlled ionization-induced injection of electrons into blowout plasma wakes in a simple experimental setup, which utilizes only the wakefields at the rear of the ion cavity to trigger the injection and trapping of electrons from a neutral atomic species into a well-defined phase of the plasma wake. As a result, high-quality electron bunches can be produced with short pulse lengths ( $\leq 1 \mu \mathrm{m} \mathrm{rms}$ ), low normalized emittances $(\sim 1 \mu \mathrm{m})$, and low uncorrelated energy spread $(<1 \%)$ on a GeV-energy scale. The first experiments demonstrating such beam quality will be regarded as important milestones in the ongoing endeavor to advance plasma-based particle accelerators for their future application in photon science and high-energy physics.

We thank the OSIRIS consortium (IST/UCLA) for access to the OSIRIS code. Special thanks for support go to J. Vieira and R. Fonseca. Furthermore, we acknowledge the grant of computing time by the Jülich Supercomputing Centre on JUQUEEN under Project No. HHH09. We would like to thank the Humboldt Foundation for financial support. 
[1] T. Tajima and J. M. Dawson, Phys. Rev. Lett. 43, 267 (1979).

[2] S. P. D. Mangles et al., Nature (London) 431, 535 (2004).

[3] C. G. R. Geddes, Cs. Toth, J. van Tilborg, E. Esarey, C. B. Schroeder, D. Bruhwiler, C. Nieter, J. Cary, and W. P. Leemans, Nature (London) 431, 538 (2004).

[4] J. Faure, Y. Glinec, A. Pukhov, S. Kiselev, S. Gordienko, E. Lefebvre, J.-P. Rousseau, F. Burgy, and V. Malka, Nature (London) 431, 541 (2004).

[5] W. P. Leemans, B. Nagler, A. J. Gonsalves, Cs. Tóth, K. Nakamura, C. G. R. Geddes, E. Esarey, C. B. Schroeder, and S. M. Hooker, Nat. Phys. 2, 696 (2006).

[6] J. Osterhoff et al., Phys. Rev. Lett. 101, 085002 (2008).

[7] N. A. M. Hafz et al., Nat. Photonics 2, 571 (2008).

[8] J. Faure, C. Rechatin, A. Norlin, A. Lifschitz, Y. Glinec, and V. Malka, Nature (London) 444, 737 (2006).

[9] A. Pak, K. A. Marsh, S. F. Martins, W. Lu, W. B. Mori, and C. Joshi, Phys. Rev. Lett. 104, 025003 (2010).

[10] A. J. Gonsalves et al., Nat. Phys. 7, 862 (2011).

[11] M. Fuchs et al., Nat. Phys. 5, 826 (2009).

[12] S. Kneip et al., Nat. Phys. 6, 980 (2010).

[13] V. Veksler, in Proceedings of the CERN Symposium on High Energy Accelerators and Pion Physics, Geneva, 1956 (CERN, Geneva, 1956), Vol. 1, p. 80.

[14] P. Chen, J. M. Dawson, R. W. Huff, and T. Katsouleas, Phys. Rev. Lett. 54, 693 (1985).

[15] I. Blumenfeld et al., Nature (London) 445, 741 (2007).

[16] M. J. Hogan et al., New J. Phys. 12, 055030 (2010).

[17] J. Vieira, S. F. Martins, V. B. Pathak, R. A. Fonseca, W. B. Mori, and L. O. Silva, Phys. Rev. Lett. 106, 225001 (2011).

[18] B. Hidding, G. Pretzler, J. B. Rosenzweig, T. Königstein, D. Schiller, and D. L. Bruhwiler, Phys. Rev. Lett. 108, 035001 (2012).
[19] F. Li et al., Phys. Rev. Lett. 111, 015003 (2013).

[20] T. Mehrling, J. Grebenyuk, F. S. Tsung, K. Floettmann, and J. Osterhoff, Phys. Rev. ST Accel. Beams 15, 111303 (2012).

[21] E. Oz et al., Phys. Rev. Lett. 98, 084801 (2007).

[22] J. B. Rosenzweig, B. Breizman, T. Katsouleas, and J. J. Su, Phys. Rev. A 44, R6189 (1991).

[23] K. V. Lotov, Phys. Rev. E 69, 046405 (2004).

[24] W. Lu, C. Huang, M. Zhou, W. B. Mori, and T. Katsouleas, Phys. Rev. Lett. 96, 165002 (2006).

[25] A. Perelomov, V. Popov, and M. Terentev, Sov. Phys. JETP 23, 924 (1966).

[26] M. V. Ammosov, N. B. Delone, and V. Krainov, Sov. Phys. JETP 64, 1191 (1986).

[27] D. L. Bruhwiler, D. A. Dimitrov, J. R. Cary, E. Esarey, W. Leemans, and R. E. Giacone, Phys. Plasmas 10, 2022 (2003).

[28] P. Sprangle, E. Esarey, and A. Ting, Phys. Rev. Lett. 64, 2011 (1990).

[29] P. Mora and T. M. Antonsen, Jr., Phys. Plasmas 4, 217 (1997).

[30] N. Kirby et al., Phys. Rev. ST Accel. Beams 12, 051302 (2009).

[31] C. E. Clayton et al., Phys. Rev. Lett. 88, 154801 (2002).

[32] C.E. Clayton et al., Phys. Rev. Lett. 105, 105003 (2010).

[33] P.-F. Hao, Y.-T. Ding, Z.-H. Yao, F. He, and K.-Q. Zhu, J. Micromech. Microeng. 15, 2069 (2005).

[34] R. A. Fonseca et al., Lect. Notes Comput. Sci. 2331, 342 (2002); R. A. Fonseca, S. F. Martins, L. O. Silva, J. W. Tonge, F. S. Tsung, and W. B. Mori, Plasma Phys. Controlled Fusion 50, 124034 (2008).

[35] E. Esarey, B. A. Shadwick, P. Catravas, and W. P. Leemans, Phys. Rev. E 65, 056505 (2002). 